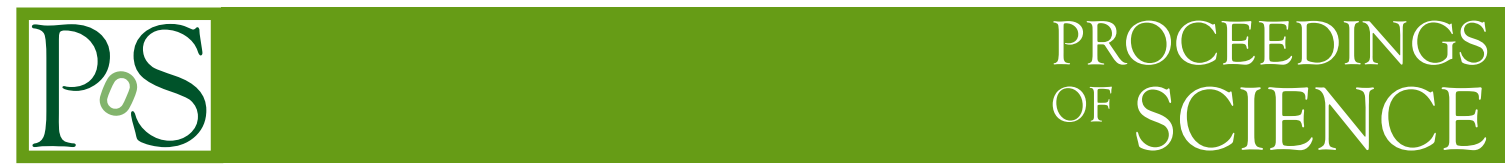

IFT-UAM/CSIC-21-118

\title{
Large triple Higgs couplings in the 2HDM at $e^{+} e^{-}$colliders
}

\section{F. Arco, ${ }^{a, b, *}$ S. Heinemeyer ${ }^{b}$ and M.J. Herrero ${ }^{a, b}$}

${ }^{a}$ Departamento de Física Teórica, Universidad Autónoma de Madrid, Cantoblanco, 28049, Madrid, Spain

${ }^{b}$ Instituto de Física Teórica (UAM/CSIC), Universidad Autónoma de Madrid, Cantoblanco, 28049, Madrid, Spain

E-mail: francisco.arco@uam.es, Sven.Heinemeyer@cern.ch, maria.herrero@uam.es

Within the framework of the $\mathcal{C P}$ conserving Two Higgs Doublet Models (2HDM) type I and II we investigate the di-Higgs production at future $e^{+} e^{-}$colliders in order to find effects coming from triple Higgs couplings. We define and explore some benchmark planes that show large values of these couplings, still in agreement with all the relevant theoretical and experimental constraints. Within those planes two production channels are considered: $e^{+} e^{-} \rightarrow h_{i} h_{j} Z$ and $e^{+} e^{-} \rightarrow h_{i} h_{j} v \bar{v}$, with $h_{i} h_{j}=h h, H H$ and $A A$. We discuss on the sensitivity to $\kappa_{\lambda}:=\lambda_{h h h} / \lambda_{h h h}^{\mathrm{SM}}$ and $\lambda_{h h H}$ in the $h h v \bar{v}$ production and to $\lambda_{h H H}\left(\lambda_{h A A}\right)$ in the $H H v \bar{v}(A A v \bar{v})$ production at CLIC $3 \mathrm{TeV}$ via the cross section distribution on the invariant mass of the final-state Higgs-pair.

*** The European Physical Society Conference on High Energy Physics (EPS-HEP2021), ***

*** 26-30 July 2021 ***

*** Online conference, jointly organized by Universität Hamburg and the research center DESY ***

${ }^{*}$ Speaker 


\section{Introduction}

One of the most popular extensions of the SM is the two Higgs doublet model (2HDM) [1]. This model leads to triple-Higgs boson interactions among the new scalars, namely $h, H, A$ and $H^{ \pm}$, which are the key focus of this contribution. Future colliders, specially $e^{+} e^{-}$colliders such as ILC [2] and CLIC [3], will play a key role in order to discover (or exclude) beyond SM physics. In this contribution, based on Refs. [4, 5], we explore the effects of the triple Higgs couplings on the production of two neutral Higgs bosons in the context of a 2HDM, type I and II, at future $e^{+} e^{-}$colliders. The two principal production channels are $e^{+} e^{-} \rightarrow h_{i} h_{j} Z$ and $e^{+} e^{-} \rightarrow h_{i} h_{j} v \bar{v}$, with $h_{i} h_{j}=h h, H H$ and $A A$. We calculate the production rate of both channels in some benchmark planes and points and analyze the sensitivity to triple Higgs couplings via the cross section distribution on the invariant mass of the final-state Higgs-pair.

\section{The Two Higgs Doublet Model (2HDM)}

The 2HDM [1] contains two Higgs doublets, $\Phi_{1}$ and $\Phi_{2}$, contrary to the SM, where only one Higgs doublet is required. In this contribution, we work in the context of a $C P$ conserving 2HDM with an imposed $Z_{2}$ symmetry, in order to avoid flavor changing neutral currents, that is only softly broken by the parameter $m_{12}^{2}$. This $Z_{2}$ symmetry leads to four different types of Yukawa sectors, where in this contribution we only consider 2HDM type I and type II. After the EW symmetry breaking, due to the new degrees of freedom, five physical states are realized: two $C \mathcal{P}$-even scalar fields, $h$ and $H$, one $C \mathcal{P}$-odd one, $A$, and one charged pair, $H^{ \pm}$

We study the 2HDM in the physical basis, where the free parameters of the model are:

$$
m_{h}, m, \tan \beta, \cos (\beta-\alpha) \equiv c_{\beta-\alpha} \text { and } m_{12}^{2},
$$

where we consider a fully degenerated scenario with $m_{H}=m_{A}=m_{H^{ \pm}} \equiv m$. The parameter $\tan \beta$ is defined as the ratio of the vevs of the two Higgs doublets, $\tan \beta:=v_{2} / v_{1}$, satisfying the relation $v_{1}^{2}+v_{2}^{2}=v^{2}$, where $v$ is the SM vev. The angles $\alpha$ and $\beta$ diagonalize the $C \mathcal{P}$-even and the $C \mathcal{P}$-odd Higgs boson sectors respectively. In our analysis we identify the lightest $C \mathcal{P}$-even Higgs boson $h$ with the one observed at $m_{h} \sim 125 \mathrm{GeV}$ and the remaining Higgs bosons are assumed to be heavier. The limit $c_{\beta-\alpha} \rightarrow 0$ is of great interest in the $2 \mathrm{HDM}$ because in that situation all SM couplings for $h$ are recovered, what is known as the alignment limit. Regarding the constraints on the model, we consider theoretical constraints, such as the tree-level perturbative unitarity and the stability of the potential, as well as experimental constraints, namely electroweak precision data, the BSM boson searches by colliders, the properties of the $125 \mathrm{GeV}$ Higgs boson and flavor observables. A detailed discussion on these constraints including all references can be found in $[4,5]$.

The key role in this contribution is played by the triple Higgs couplings $\lambda_{h_{i} h_{j} h_{k}}$. We define these couplings such that the Feynman rule of the interaction $h_{i} h_{j} h_{k}$ is given by $-i v n ! \lambda_{h_{i} h_{j} h_{k}}$, where $n$ is the number of identical particles in the vertex. We furthermore define $\kappa_{\lambda}:=\lambda_{h h h} / \lambda_{h h h}^{\mathrm{SM}}$. The allowed range of triple Higgs couplings involving at least one SM-like Higgs boson by all the relevant theoretical and experimental constraints can be found in [4]. 

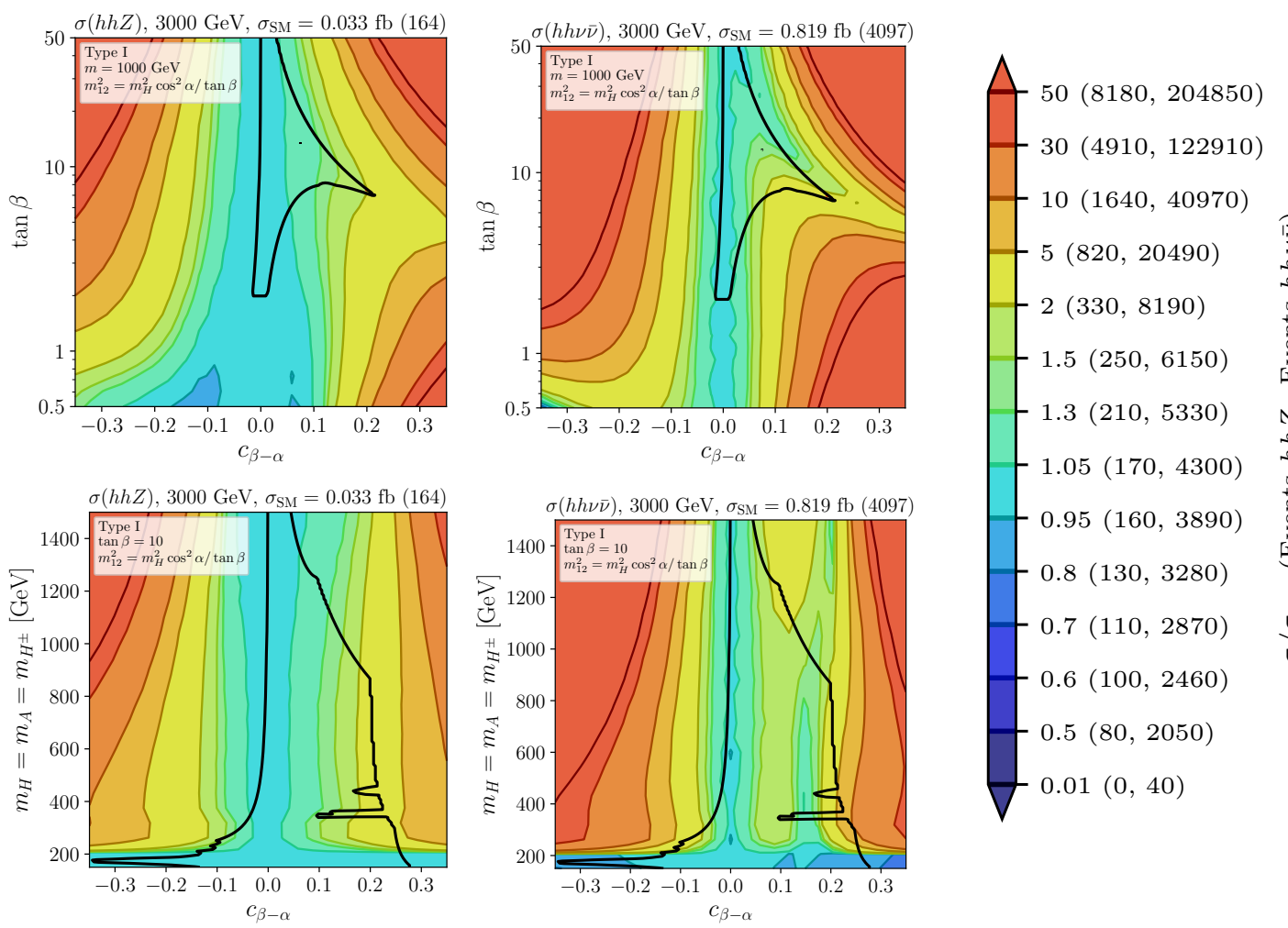

Figure 1: Cross sections for $e^{+} e^{-} \rightarrow h h Z$ (left) and $e^{+} e^{-} \rightarrow h h v \bar{v}$ (right) relative to the SM at $\sqrt{s}=3 \mathrm{TeV}$ for the benchmark planes $\left(c_{\beta-\alpha}, \tan \beta\right)$ in the upper row and $\left(c_{\beta-\alpha}, m\right)$ in the lower row.

\section{3. $h h$ production in $2 \mathrm{HDM}$ type I}

The cross section predictions presented in this contribution are calculated at tree-level with the help of the public codes MadGraph5 [6], FeynRules [7], 2HDMC [8] and R00T [9]. Electrons and positrons were assumed to be massless and all diagrams are included in the computation. Here we only consider production at $\sqrt{s}=3 \mathrm{TeV}$ (the highest energy potentially reachable at CLIC) with an expected luminosity of $\mathcal{L}_{\text {int }}=5 \mathrm{ab}^{-1}$ [3]. For other energies see [5].

In Fig. 1 we show the cross section predictions w.r.t. the SM prediction for $e^{+} e^{-} \rightarrow h h Z$ and $e^{+} e^{-} \rightarrow h h v \bar{v}$ in the following benchmark planes in the 2HDM type I: plane $\left(c_{\beta-\alpha}, \tan \beta\right)$, with $m=1 \mathrm{TeV}$ and $m_{12}^{2}=m_{H} \cos ^{2} \alpha / \tan \beta$, and plane $\left(c_{\beta-\alpha}, m\right)$, with $\tan \beta=10$ and $m_{12}^{2}=$ $m_{H} \cos ^{2} \alpha / \tan \beta$. At this energy the neutrino channel has a much larger cross section than the $Z$ channel. In the $\left(c_{\beta-\alpha}, \tan \beta\right)$ plane (upper plots), cross sections around $2 \sigma_{\mathrm{SM}}$ can be found inside the allowed region (enclosed by the black solid line). On the other hand, in the $\left(c_{\beta-\alpha}, m\right)$ plane (lower plots) a cross section $\sim 3 \sigma_{\mathrm{SM}}$ can be realized for a wide range of heavy Higgs bosons masses within the allowed region. Furthermore, $h h v \bar{v}$ production rates up to $10 \sigma_{\mathrm{SM}}$ can be found inside the allowed region for very low $m$ and large $c_{\beta-\alpha}$. In order to find sensitivity to triple Higgs couplings we propose the study of the cross section distributions on the invariant mass of the final-state Higgspair. In Fig. 2, we present the $\mathrm{d} \sigma / \mathrm{d} m_{h h}$ distribution for $e^{+} e^{-} \rightarrow h h v \bar{v}$ of four specific benchmark points (BPs), whose input parameters are given in Tab. 1. This set of BPs satisfies all the relevant 

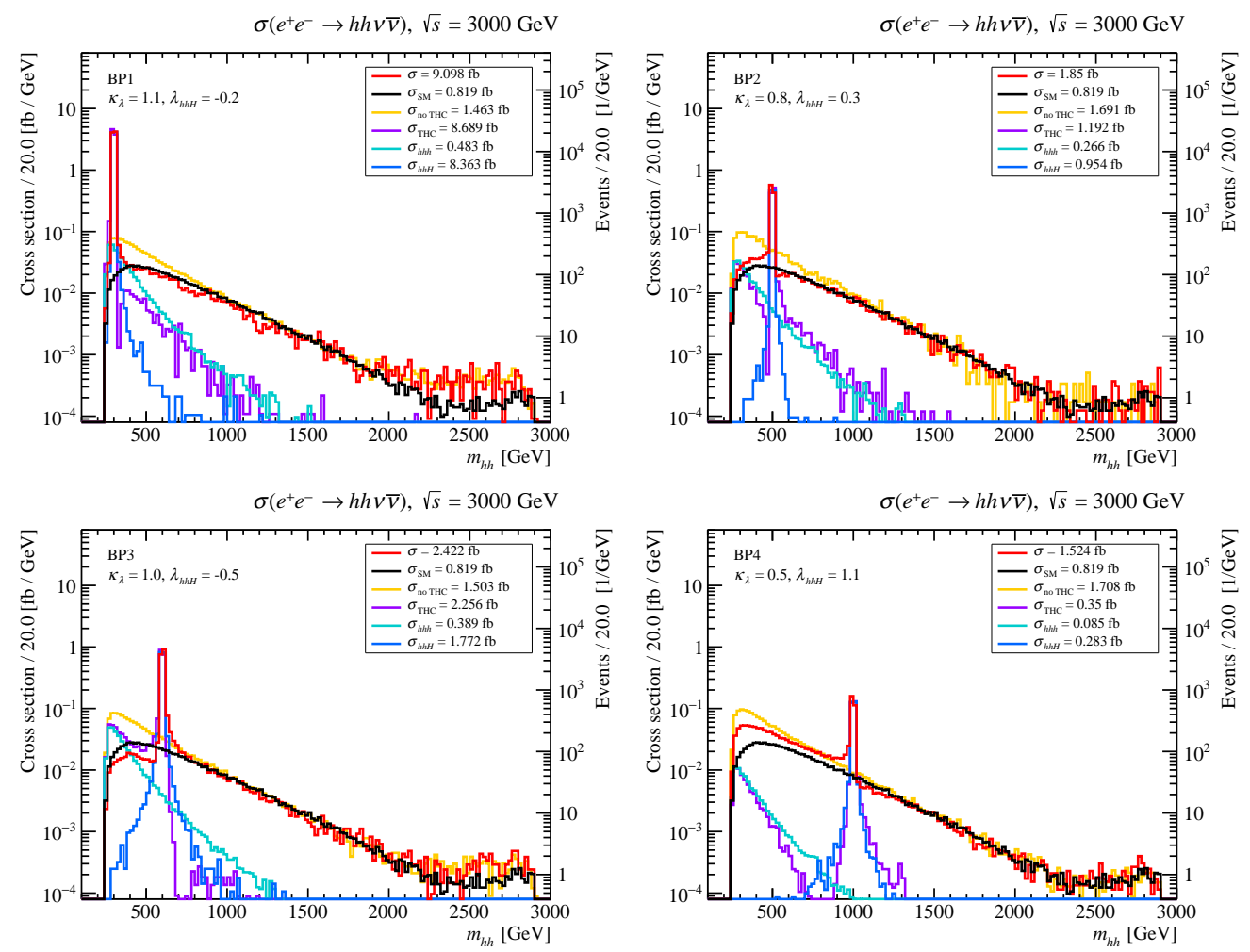

Figure 2: Distribution on the invariant mass of the final-state $h h$ pair in the process $e^{+} e^{-} \rightarrow h h v \bar{v}$ at $\sqrt{s}=3 \mathrm{TeV}$ for BP1, BP2, BP3 and BP4 defined in Tab. 1.

\begin{tabular}{c|c|c|c|c|c|c|c|c|c|c} 
Point & $m$ & $\tan \beta$ & $c_{\beta-\alpha}$ & $m_{12}^{2}$ & $\kappa_{\lambda}$ & $\lambda_{h h H}$ & $\lambda_{h H H}$ & $\lambda_{H H H}$ & $\Gamma_{H}$ & $\Gamma_{A}$ \\
\hline BP1 & 300 & 10 & 0.25 & $m_{H} \cos ^{2} \alpha / \tan \beta$ & 1.1 & -0.2 & 2 & 0.3 & 0.84 & 0.18 \\
\hline BP2 & 500 & 7.5 & 0.1 & 32000 & 0.8 & 0.3 & 2 & 0.6 & 0.88 & 0.71 \\
\hline BP3 & 600 & 10 & 0.2 & $m_{H} \cos ^{2} \alpha / \tan \beta$ & 1.0 & -0.5 & 6 & 0.6 & 5.1 & 2.6 \\
\hline BP4 & 1000 & 8.5 & 0.08 & $m_{H} \cos ^{2} \alpha / \tan \beta$ & 0.5 & 1.1 & 6 & -0.2 & 5.8 & 2.6
\end{tabular}

Table 1: Benchmark points in the 2HDM type I (masses and widths are given in $\mathrm{GeV}$ ).

constraints, covers a wide range on the heavy Higgs bosons masses and leads to sizable triple Higgs couplings. It can be seen in Fig. 2 that the effect from $\kappa_{\lambda}$ (light blue lines) for all BPs appears close to the threshold region at $m_{h h}=250 \mathrm{GeV}$, similar to what happens in the SM. Therefore, we expect that the sensitivity to $\kappa_{\lambda}$ to be comparable to that obtained for future $e^{+} e^{-}$colliders in the context of the SM [10]. Furthermore, from the comparison of the distributions of the total cross section (red lines) and the contribution from diagrams without triple Higgs couplings (yellow lines) that close to threshold region one can deduce that diagrams with $\kappa_{\lambda}$ have a destructive interference with the diagrams without triple Higgs couplings in the $h h v \bar{v}$ production, just as in the SM. The sensitivity to $\lambda_{h h H}$ enters through $H$ mediated diagrams (dark blue lines) that can produce a resonant peak around $m_{h h}=m_{H}$, as it can be seen in all BPs in Fig. 2. We propose a theoretical estimator $R$ to study the sensitivity to $\lambda_{h h H}$ via the $h \rightarrow b \bar{b}$ decays defined as:

$$
R=\frac{\bar{N}^{R}-\bar{N}^{C}}{\sqrt{\bar{N}^{C}}} \text {, with } \bar{N}=N \times \mathcal{A} \times\left(\epsilon_{b}\right)^{4},
$$



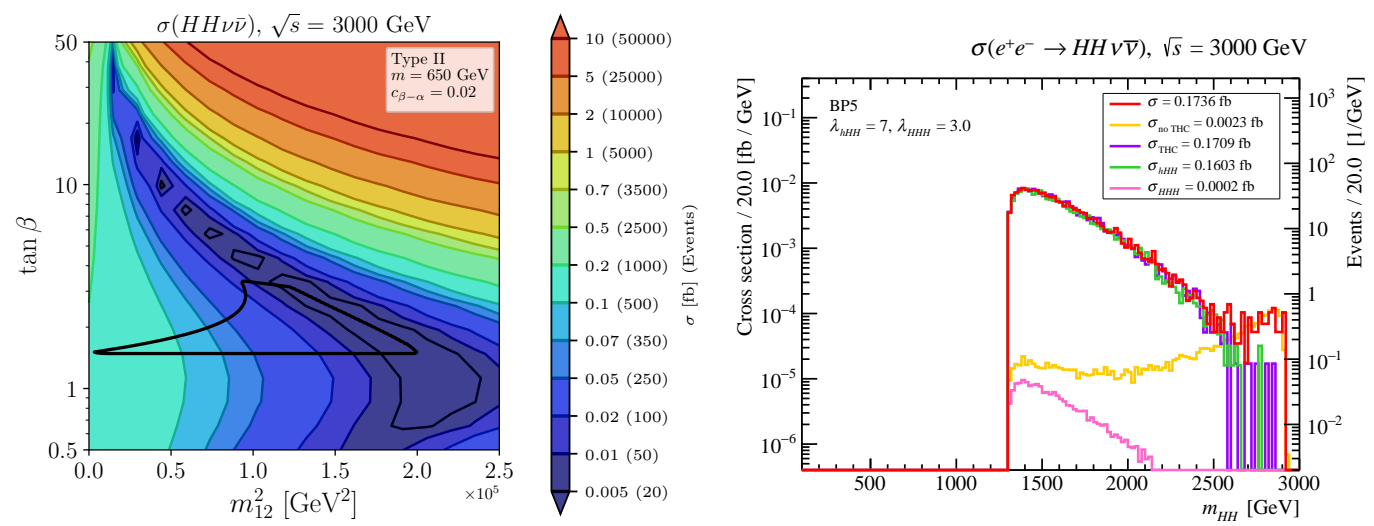

Figure 3: Cross section for $e^{+} e^{-} \rightarrow H H v \bar{v}$ (left) and distribution on the invariant mass of the final-state $H H$ pair for BP5 (right) at $\sqrt{s}=3 \mathrm{TeV}$.

where $N$ is the number of final four $b$-jets events nearby the resonant peak, the superscript $R, C$ refers to events coming from the $H$ mediated resonant diagrams and from the diagrams with no triple Higgs couplings, respectively. $\epsilon_{b}$ is the $b$-tagging efficiency of the detector, that we considered around $80 \%$, and $\mathcal{A}$ is the detection acceptance assuming the following cuts (see [5] for details):

$$
p_{T}^{b}>20 \mathrm{GeV} ; \quad\left|\eta^{b}\right|<2 ; \quad \Delta R_{b b}>0.4 ; \quad p_{T}^{Z}>20 \mathrm{GeV} ; \quad E_{T}^{\prime}>20 \mathrm{GeV} .
$$

We find larger values of $R$ for the $h h v \bar{v}$ production channel than for $h h Z$ at all studied center of mass energies, except for BP1 at $\sqrt{s}=500 \mathrm{GeV}$ [5]. Moreover, the larger values of $R$ are found for $h h v \bar{v}$ at $3 \mathrm{TeV}$. Therefore, CLIC $3 \mathrm{TeV}$ is the best suited collider to access to $\lambda_{h h H}$ according to our estimator $R$. The precise values for $\bar{N}^{R / C}, \mathcal{A}$ and $R$ for all BPs for both $h h Z$ and $h h v \bar{v}$ can be found in [5].

\section{4. $H H v \bar{v} \sim A A v \bar{v}$ production in 2HDM type II}

In the left panel of Fig. 3 we show the production cross section of $H H v \bar{v}$ at $3 \mathrm{TeV}$ in the plane $\left(m_{12}^{2}, \tan \beta\right.$ ) with $m=650 \mathrm{GeV}$ and $c_{\beta-\alpha}=0.02$, in 2HDM type II. In the degenerated scenario where $m_{H}=m_{A}$ one finds $\sigma(H H v \bar{v}) \simeq \sigma(A A v \bar{v})$, so we only show the former. It can be seen that the cross section reaches its maximum value, close to $0.2 \mathrm{fb}$, within the allowed region (delimited by the solid black line) for $m_{12}^{2} \lesssim 5 \times 10^{5} \mathrm{GeV}^{2}$. This maximum coincides with the largest values for $\lambda_{h h H}$ in that plane, where couplings around 7 are realized. On the other hand, in the left panel of Fig. 3 we show the differential cross section distribution of $e^{+} e^{-} \rightarrow H H v \bar{v}$ as a function of $m_{H H}$ for the point $\tan \beta=1.5$ and $m_{12}^{2}=10000 \mathrm{GeV}^{2}$ within the previously discussed plane (which is denoted as BP5). One can see that the effect coming from the diagrams depending on $\lambda_{h H H}$ (green lines) dominates over the rest of the contributions and is nearly fully responsible for the total cross section. This indicates that the total cross section can receive a relevant contribution from the diagrams containing $\lambda_{h H H}$, in particular from the non-resonant contributions, which dominate at this large center-of-mass energy. It is worth noticing that this situation can be reproduced in the 2HDM type I if a similar value of $\lambda_{h H H}$ is realized. 


\section{Summary and conclusions}

In this contribution, based on Refs. [4, 5], we show the di-Higgs production cross sections at future high energy $e^{+} e^{-}$colliders in the 2HDM type I and II. Here we focus on a center-of mass energy of $3 \mathrm{TeV}$, the projected final energy state of CLIC. We consider two different channels, $e^{+} e^{-} \rightarrow h_{i} h_{j} Z$ and $e^{+} e^{-} \rightarrow h_{i} h_{j} v \bar{v}$ with $h_{i} h_{j}=h h, H H, A A$. We analyze the production rate of these processes within several benchmark planes that present sizable values for triple Higgs couplings while being in agreement with all theoretical and experimental constraints. By means of the cross section distributions on $m_{h_{i} h_{j}}$, we find sizable effects from $\kappa_{\lambda}$ and $\lambda_{h h H}$ in the $h h v \bar{v}$ production and effects from $\lambda_{h H H}$ and $\lambda_{h A A}$ in the $H H v \bar{v}$ and $A A v \bar{v}$ production respectively.We find a dependence on $\kappa_{\lambda}$ similar to the one present in the SM, where the largest influence on the cross section happens on the region slightly above $m_{h h}=250 \mathrm{GeV}$. On the other hand, the dependence on $\lambda_{h h H}$ enters through resonant diagrams mediated by the $H$ boson. We found sensitivity to this resonance (and in consequence to $\lambda_{h h H}$ ) for a wide range of masses of the BSM heavy Higgs bosons. Via a theoretical estimator $R$, that takes into account the detection of the final four $b$-jet state, we conclude that CLIC $3 \mathrm{TeV}$ will be the most sensitive future $e^{+} e^{-}$collider to $\lambda_{h h H}$. Finally, we find that $H H v \bar{v}(A A v \bar{v})$ can be completely dominated by the value of $\lambda_{h H H}\left(\lambda_{h A A}\right)$, reaching production rates up to $0.2 \mathrm{fb}$. That would mean that an experimental detection of these channels would provide an interesting opportunity to measure $\lambda_{h H H}$ and $\lambda_{h A A}$ at CLIC $3 \mathrm{TeV}$.

\section{References}

[1] G. C. Branco, P. M. Ferreira, L. Lavoura, M. N. Rebelo, M. Sher and J. P. Silva, Phys. Rept. 516 (2012) 1 [arXiv:1106.0034 [hep-ph]].

[2] P. Bambade et al. [arXiv:1903.01629 [hep-ex]].

[3] P. N. Burrows et al. [CLICdp and CLIC], [arXiv:1812.06018 [physics.acc-ph]].

[4] F. Arco, S. Heinemeyer and M. J. Herrero, Eur. Phys. J. C 80 (2020) no.9, 884 [arXiv:2005.10576 [hep-ph]].

[5] F. Arco, S. Heinemeyer and M. J. Herrero, Eur. Phys. J. C 81 (2021) no.10, 913 [arXiv:2106.11105 [hep-ph]].

[6] J. Alwall, R. Frederix, S. Frixione, V. Hirschi, F. Maltoni, O. Mattelaer, H. S. Shao, T. Stelzer, P. Torrielli and M. Zaro, JHEP 07 (2014), 079 [arXiv:1405.0301 [hep-ph]].

[7] A. Alloul, N. D. Christensen, C. Degrande, C. Duhr and B. Fuks, Comput. Phys. Commun. 185 (2014) 2250 [arXiv:1310.1921 [hep-ph]].

[8] D. Eriksson, J. Rathsman and O. Stål, Comput. Phys. Commun. 181 (2010) 189 [arXiv:0902.0851 [hep-ph]].

[9] R. Brun and F. Rademakers, Nucl. Instrum. Meth. A 389 (1997), 81-86

[10] P. Roloff et al. [CLICdp], Eur. Phys. J. C 80 (2020) no.11, 1010 [arXiv:1901.05897 [hep-ex]]. 